\title{
Massnahmen zur Verbesserung der Gesundheit des Bewegungsapparates*
}

\author{
Im Rahmen des Nationalen Forschungsprogramms NFP 53 wurde zwischen April \\ 2004 und März 2009 in zahlreichen Projekten die Gesundheit des Bewegungsapparats \\ der Schweizer Bevölkerung untersucht. Der Beitrag schildert die wichtigsten \\ Erkenntnisse und Forderungen zur Verbesserung der Versorgungsqualität. Es mache \\ jedoch wenig Sinn, die muskuloskelettale Gesundheit isoliert zu betrachten.
}

\author{
Mathis Brauchbara, \\ Françoise Allaz ${ }^{b}$, \\ Bettina Schultec, \\ Regine Strittmatter ${ }^{\text {, }}$ \\ Andreas Stucke \\ a Umsetzungsbeauftragter \\ NFP 53, advocacy AG \\ b Prof. Dr. med., Mitglied des \\ Vorstands der SAMW, \\ Universitätsspital Genf \\ c Vizedirektorin \\ Gesundheitsförderung \\ Schweiz \\ d stv. Geschäftsleiterin \\ Rheumaliga Schweiz \\ e Prof. Dr. med., Präsident der \\ Leitungsgruppe des NFP 53, \\ Inselspital Bern
}

* Kurzfassung des White Papers «Förderung der Gesundheit des Bewegungsapparates - Ein Gewinn für Betroffene und Gesellschaft», zu beziehen auf der Website des NFP 53
Im Jahr 2003 hat der Bundesrat den Schweizerischen Nationalfonds damit beauftragt, ein Nationales Forschungsprogramm zur Gesundheit des Bewegungsapparates durchzuführen. Er entschied sich für diese Fragestellung, weil die Schweizer Bevölkerung in hohem Masse von Beschwerden und Krankheiten des Bewegungsapparats betroffen ist: Fast alle Menschen haben irgendwann im Verlauf ihres Lebens eine muskuloskelettale Erkrankung.

Diese Erkrankungen belasten die gesamte Volkswirtschaft mit hohen Kosten: In den Betrieben führen muskuloskelettale Leiden zu Absenzen, die jährlich betriebliche Kosten von 0,97 Milliarden Franken verursachen. Hinzu kommt die verlorene Produktionsleistung in der Höhe von jährlich rund 3,3 Milliarden Franken [1]. Ein Fünftel aller Renten werden vor allem wegen chronischer Rückenbeschwerden gesprochen [2]. Alleine in der Invalidenversicherung entstehen so jährliche Kosten von rund einer Milliarde Franken. In den kommenden Jahren ist zudem mit einer starken Zunahme muskuloskelettaler Erkrankungen und der daraus resultierenden Kosten $\mathrm{zu}$ rechnen. Es ist daher dringend notwendig, Strategien umzusetzen, die darauf abzielen, die Krankheitslast zu mindern. Die Leitungsgruppe des Nationalen Forschungsprogramms NFP 53, die Akademie der Medizinischen Wissenschaften, die Rheumaliga Schweiz und Gesundheitsförderung Schweiz haben deshalb ein «White Paper» entwickelt, das Schlüsse aus dem NFP 53 und dem aktuellen, internationalen Stand des Wissens zieht [3].

\section{Prävention besser koordinieren}

Die Gesundheit des Bewegungsapparats betrifft neben dem Gesundheitswesen auch das Sozialwesen, die Arbeitswelt, den Verkehr, die Freizeit oder den Städtebau. Muskuloskelettale Gesundheit ist daher Aufgabe des gesamten Staats- und Gemeinwesens. In den letzten Jahrzehnten wurden genügend Erkenntnisse gewonnen, welche die Wirksamkeit der Prävention gewisser muskuloskelettaler Erkrankungen belegen.

\section{Mesures à prendre pour améliorer} la santé de l'appareil locomoteur

Le programme national de recherche PNR 53 a montré qu'il est nécessaire de mettre en œuvre des mesures de prévention, de traitement et de réadaptation pour diminuer la charge de morbidité liée aux maladies musculosquelettiques. Les programmes de prévention déjà mis en place par la Confédération, les cantons et l'économie privée doivent être mieux coordonnés et plus axés sur les maladies de l'appareil locomoteur. La prévention en entreprise devrait quant à elle mettre davantage l'accent sur les maladies chroniques, notamment les maladies de l'appareil locomoteur.

Pour améliorer la qualité des soins pour les personnes souffrant de ces maladies il faut continuer à développer des directives de traitement et veiller à ce que prestataires de soins les appliquent plus systématiquement. Pour ce faire les autorités régulatrices et les instances financières doivent $s$ entendre sur les directives et sur leur financement. Par ailleurs, il est essentiel que les patients prennent une part active dans leur traitement et dans les mesures de réadaptation afin d'en améliorer l'efficacité. Pour mettre à profit ce potentiel encore sousexploité, les patients doivent être mieux formés et les mesures indicatives pour les prestataires mieux définis. Enfin, la recherche portant sur l'optimisation de la prise en charge - aussi bien en ce qui concerne les soins que la réadaptation - des personnes touchées par ces maladies devraient se concentrer davantage sur les traitements non-médicamenteux (moyens auxiliaires, soins, physiothérapie, ergothérapie, programmes de réadaptation). 


\begin{abstract}
NFP 53 - Forschung zur muskuloskelettalen Gesundheit

Im Rahmen des Nationalen Forschungsprogramms NFP 53 haben zwischen April 2004 und März 2009 insgesamt 26 verschiedene Forschungsprojekte die Gesundheit des Bewegungsapparats ausgeleuchtet. Dabei haben Forschende die Ursachen der Beschwerden untersucht, die bestehenden Therapien kritisch hinterfragt und neue Ansätze entwickelt, die helfen, die Gesundheit des Bewegungsapparates aufrechtzuerhalten oder wiederherzustellen. Die Resultate der Projekte und des Programms als Ganzes sind auf der Website des Programms erhältlich (www.nfp53.ch).
\end{abstract}

Auch mehrere Projekte des NFP 53 konnten neue Hinweise für die Wirksamkeit präventiver und gesundheitsförderlicher Massnahmen liefern.

Auf der Basis bestehender Evidenz lassen sich allgemeine Empfehlungen, etwa im Bereich der Bewegung oder der Ernährung, ableiten. Die meisten Emp-

\section{«Die Gesundheitsförderung sollte am Arbeitsplatz}

\section{einen höheren Stellenwert erhalten.»}

fehlungen zielen aber nicht alleine auf die muskuloskelettale Gesundheit allein ab, sondern auch auf andere gesundheitliche Aspekte wie etwa die Gesundheit des Herz-Kreislauf-Systems, die Krebsprävention oder die Vorbeugung von Diabetes. Aus diesem Grund macht es wenig Sinn, die muskuloskelettale Gesundheit isoliert zu betrachten oder gar breite, nationale Programme zu fordern: Bereits existierende Gesundheitsprogramme und -ansätze helfen, die Gesundheit des Bewegungsapparats zu fördern. Dazu gehören das Nationale Programm Ernährung und Bewegung, die Kantonalen Aktionsprogramme Gesundes
Körpergewicht, das Nationale Präventionsprogramm Tabak oder das Nationale Präventionsprogramm Alkohol. Diese Ansätze müssen aber harmonisiert und unter Berücksichtigung der Gesundheit des Bewegungsapparates optimiert werden.

Wenn Prävention in der Schweiz in der Vergangenheit oft ungenügend effizient war, dann auch weil die Präventionsaktivitäten zu sehr aus dem Blickwinkel der Prävention einzelner Krankheiten betrachtet wurden und nicht auf der Basis zu erreichender Gesundheitsziele. Das Präventionsgesetz bietet nun die Gelegenheit, bestehende Präventionsaktivitäten auf Basis gemeinsamer Gesundheitsziele sachgerecht zu harmonisieren. Bei der Formulierung der Präventionsziele muss aber - schon alleine aufgrund der hohen Krankheitslast und der volkswirtschaftlichen Kosten - die Gesundheit des Bewegungsapparates adäquat berücksichtigt werden. Darüber hinaus sollten die Präventionsziele so gesetzt werden, dass Prävention und Gesundheitsförderung jenen Personengruppen zugute kommen, die am stärksten behindert sind oder die am stärksten sozial benachteiligt sind.

Die Arbeitswelt verändert sich im Zuge der Globalisierung und durch die demographischen Trends: Die Arbeitsbelastung nimmt zu, von den Arbeitnehmenden wird eine höhere Flexibilität gefordert und es ist damit zu rechnen, dass der Anteil älterer Männer und Frauen in den Betrieben stark zunehmen wird. Es ist zudem bekannt, dass Stress im Zusammenhang mit Krankheiten des Bewegungsapparates zu Dauerschäden führen kann. Die Gesundheitsförderung sollte daher am Arbeitsplatz einen höheren Stellenwert erhalten. Sie macht schon alleine deshalb Sinn, weil der finanzielle Nutzen für einen Betrieb gemäss einschlägigen internationalen Studien höher ist als die Ausgaben für die Gesundheitsförderung [4]. Massnahmen sind auf mehreren Ebenen notwendig:

- Um die betriebliche Gesundheitsförderung besser zu etablieren, müsste sie besser erforscht werden.

- Anreizsysteme sollten eingeführt werden, die es den Betrieben erleichtern, in der betrieblichen Gesundheitsförderung aktiv zu werden. Dies kann beispielsweise über die betrieblichen Versicherungen erfolgen.

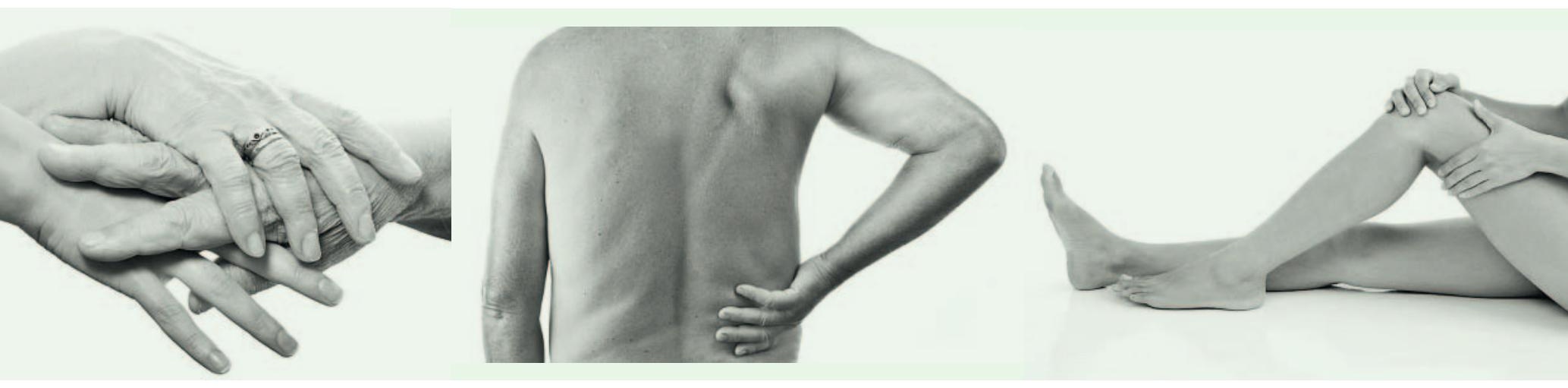


- Bisher war die Prävention am Arbeitsplatz stark auf die Unfallprävention ausgerichtet. Dies sollte sich angesichts der Zunahme chronischer, nicht unfallbedingter Erkrankungen ändern, was aber wiederum gesetzliche Anpassungen etwa im Unfallversicherungsgesetz oder im Arbeitsgesetz nötig macht.

Neben allgemeinen Empfehlungen für die gesamte Bevölkerung gilt es, sich vermehrt auf Zielgruppen mit spezifischen Risiken zu konzentrieren. Dies können Kinder und Jugendliche sein, die sich etwa im Sport besonderen Gefahren aussetzen oder Angestellte, die an Arbeitsplätzen arbeiten, die häufig mit Problemen des Bewegungsapparates einhergehen. Bei

\section{«Im Hinblick auf Richtlinien besteht bei Arthrose und Rehabilitation im Alter noch Nachholbedarf.»}

Betagten stehen unter anderem die Risiken von Stürzen mit Knochenbrüchen im Vordergrund. Bereits heute gibt es kostengünstige Methoden, wie ältere Personen mit einem erhöhten Risiko für Knochenbrüche gezielt identifiziert und im Hinblick auf die Prävention beraten und begleitet werden können. Doch das Potential dieser Interventionsformen wird in der Schweiz noch ungenügend genutzt.

\section{Behandlung optimieren}

Untersuchungen der letzten Jahre zeigen, dass die Qualität der medizinischen Versorgung in der Schweiz von Region zu Region stark verschieden ist. Dies betrifft auch Krankheiten des Bewegungsapparates. Diese starke Ungleichheit verweist auf grosse Unterschiede in der Versorgungsqualität innerhalb des Gesundheitssystems.

Wenn grosse qualitative Unterschiede in der Behandlung bestehen, sollten allgemein gültige Richtlinien helfen, die Qualität zu sichern. Solche Guidelines sollten dazu beitragen, klinische Evidenz in die medizinische Praxis zu überführen und dadurch die Versorgung der Bevölkerung zu verbessern. Für die Entwicklung dieser Richtlinien sind die Schweizer Fachgesellschaften und medizinischen Verbände verantwortlich. Die Schweizerische Gesellschaft für Rheumatologie und internationale Organisationen (European League Against Rheumatism EULAR, American College of Rheumatology ACR, British Society for Rheumatology BSR) haben bereits zahlreiche Behandlungsrichtlinien und Therapieempfehlungen erarbeitet. Während die bestehenden Behandlungsrichtlinien das Thema Rückenschmerzen relativ umfassend behandeln, besteht bei der Arthrose und der Rehabilitation im Alter jedoch noch Nachholbedarf.
Doch Richtlinien, die nicht angewendet werden, sind nutzlos: Die Aus- und Weiterbildung der Leistungserbringer muss sich auch an diesen Richtlinien orientieren. Die Anreize im Gesundheitssystem sollten vermehrt so gesetzt werden, dass diese Richtlinien und damit die korrekte Behandlung auch im Praxisalltag umgesetzt werden. Um die Qualität der Behandlung zu fördern, sollten die Leistungserbringer auch erheben, wie sie behandeln und ob diese Richtlinien eingehalten werden. Letztlich muss es das Ziel sein, dass möglichst allen Patientinnen und Patienten eine adäquate Behandlung zukommt und dass Über- oder Unterversorgung weitgehend vermieden werden können.

Angesichts der wachsenden Zahl muskuloskelettaler Behandlungen und der steigenden Kosten im Gesundheitswesen nimmt der Druck zu, nur noch solche Interventionen durch die obligatorische Krankenpflegeversicherung $\mathrm{zu}$ finanzieren, für deren Wirksamkeit klare Belege (Evidenz) vorliegen. In vielen Bereichen fehlt aber dieser Wirksamkeitsnachweis, was auch dadurch bedingt ist, dass es für bereits etablierte Methoden wenig Anreiz für Forschung gibt. Die Erfahrungen der letzten Jahre belegen denn auch, dass unabhängige Forschung im Bereich der Behandlung der Beschwerden des Bewegungsapparates schwer zu finanzieren ist.

Öffentlich finanzierte Forschung sollte vor allem dort ansetzen, wo die privat finanzierte, industrielle Forschung nicht oder ungenügend tätig ist. Die Wirksamkeitsforschung sollte sich daher stärker auf nicht medikamentöse Behandlungsformen konzentrieren, zumal diese bei muskuloskelettalen Beschwerden wichtige Interventionen darstellen (Physiotherapie, Ergotherapie, Pflege, Hilfsmittel).

Das Potential der Selbstbeteiligung der Patientinnen und Patienten in der Behandlung wird noch $\mathrm{zu}$ wenig genutzt und sollte auf mehreren Ebenen gefördert werden: Die Motivation der Patientinnen und Patienten sollte in der Arztpraxis einen hohen Stellenwert erhalten. Im Praxisalltag ist dieser Anspruch derzeit aber schwer umzusetzen. Die Anreize - auch finanzieller Art - sollten daher so gesetzt werden, dass das motivierende Gespräch mit den Betroffenen attraktiv und gefördert wird. Niederschwellige Angebote zur Patientenbildung sollten darauf ausgerichtet sein, auch jene Personen zu erreichen, die über ein geringes Gesundheitswissen verfügen (Health Literacy). Bereits existierende Angebote von Gesundheitsorganisationen sollten sich vermehrt an diesen Zielgruppen ausrichten und sollten von Bund und Kantonen gefördert werden.

\section{Rehabilitation effizienter gestalten}

Die Förderung der Gesundheit des Bewegungsapparats konzentriert sich bei Erwachsenen im Erwerbsalter vor allem darauf, ihre Arbeitsfähigkeit möglichst umfassend zu erhalten. Um das Potential des betrieblichen Gesundheitsmanagements besser nutzen zu 
können, sind Massnahmen auf mehreren Ebenen notwendig:

- In der medizinischen Ausbildung an den Universitäten wie auch in den Ausbildungen zu Gesundheitsberufen (Ergotherapie, Pflege, Physiotherapie) sollte die Arbeitsmedizin stärker als bisher berücksichtigt werden.

- Die Unternehmen, Grossbetriebe wie auch kleinere und mittlere Unternehmen (KMUs) sollten das betriebliche Gesundheitsmanagement im Rahmen ihrer unternehmerischen Verantwortung besser verankern.

- Die Versicherer sollten durch geeignete Anreizstrukturen in ihren Versicherungsmodellen das betriebliche Gesundheitsmanagement fördern.

Es reicht nicht, chronische Verläufe am Arbeitsplatz frühzeitig zu erkennen. Vielmehr gilt es, den Betroffenen angemessene und wirksame Massnahmen anzubieten, damit diese möglichst rasch und möglichst vollständig ihre Arbeit wieder aufnehmen können. Neben den stationären Rehabilitationseinrichtungen sind in der Schweiz vermehrt auch ambulante Angebote notwendig. Dies ermöglicht eine Form der Rehabilitation, die einen starken Bezug zur Arbeitswelt hat. Sie vermindert das Risiko deutlich, dass ein Arbeitnehmer seine Arbeitsfähigkeit permanent verliert.

\section{Massnahmen dringlich umsetzen}

Die Verbesserung der Prävention, der Behandlung und der Rehabilitation von Erkrankungen des Bewegungsapparats ist dringlich, denn die Krankheitslast und die volkswirtschaftlichen Folgen wiegen schwer. Die Mittel und Möglichkeiten, um die Häufigkeit, den Schweregrad und die Folgen von muskuloskelettalen Erkrankungen zu vermindern, sind vorhanden. Aufgerufen die notwendigen Massnahmen zu ergreifen sind einerseits Ärzte, Physiotherapeuten, Pflegende, Präventionsfachleute aber auch die Arbeitgeber. Gefordert sind aber auch die Betroffenen selbst. Sie sollten sich vermehrt aktiv an ihrer Rehabilitation und Heilung beteiligen und so die Chancen eines positiven Verlaufes ihrer Beschwerden erhöhen.

Andererseits sind Massnahmen auf der Ebene des Gesundheitssystems und der Wirtschaft notwendig. Gesetzgeber, Verwaltungen bei Bund und Kantonen, aber auch die Versicherer müssen Rahmenbedingungen und Anreize schaffen, welche die muskuloskelettale Gesundheit fördern und die Effizienz der Behandlung verbessern.

Neben der Umsetzung des Wissens in die Praxis braucht es aber auch weiterhin Forschung. Sie muss dazu beitragen, der Schweizer Bevölkerung neue Formen der Prävention oder neue Therapieverfahren zu ermöglichen. Gleichzeitig muss sie dazu beitragen, die Effizienz und die Qualität im Bereich der Prävention, der Behandlung und der Rehabilitation wesentlich zu steigern.

\section{Literatur}

1 Staatssekretariat für Wirtschaft seco; 2009.

2 IV-Statist. Bundesamt für Sozialversicherungen; 2008.

3 u. a. European Action Towards Better Musculoskeletal Health 2005. The Bone and Joint Decade.

4 u. a. Gesundheitsförderung Schweiz. Studien zur Gesundheitsförderung. Bern; 2010. 